\title{
Performance analysis of impulsive noise blanking for multi-carrier PLC systems
}

\author{
Tomoya Kageyama ${ }^{\text {a) }}$ and Osamu Muta ${ }^{\text {b) }}$ \\ Kyushu University, Motooka, Fukuoka, Japan \\ a)kageyama@mobcom.ait.kyushu-u.ac.jp \\ b)muta@ait.kyushu-u.ac.jp,muta@m.ieice.org
}

\begin{abstract}
In powerline communication systems (PLC), suppression of impulsive noise is a challenging problem. One of the existing methods to mitigate impulsive noise is a deliberate blanking which removes the received samples that exceed a given threshold. However, if the received signal amplitude exceeds the blanking threshold, it may cause miss-detection of impulsive noise. Therefore, it is important to determine the blanking threshold properly. In this article, we theoretically analyze the impact of the blanking threshold selection on achievable performance, i.e., probability of impulsive noise detection (PoD), probability of false alarm (PoF), and bit error rate (BER) in multi-carrier PLC systems.
\end{abstract}

Keywords: powerline communications (PLC), impulsive noise, blanking, probability of detection $(\mathrm{PoD})$, probability of false alarm $(\mathrm{PoF})$

Classification: Wireless Communication Technologies

\section{References}

[1] W. Y. Chen, Home Networking Basis: Transmission Environments and Wired/ Wireless Protocols 2003.

[2] Y. H. Ma, P. L. So, and E. Gunawan, "Performance analysis of OFDM systems for broadband power line communications under impulsive noise and multipath effects," IEEE Trans. Power Deliv., vol. 20, no. 2, pp. 674-682, Apr. 2005. DOI:10.1109/TPWRD.2005.844320

[3] K. M. Rabie and E. Alsusa, "Effective noise cancellation using single-carrier FDMA transmission in power-line channels," IEEE Trans. Power Deliv, vol. 29 , no. 5, pp. 2110-2117, Oct. 2014. DOI:10.1109/TPWRD.2013.2297232

[4] S. A. Bhatti, Q. Shan, I. A. Glover, R. Atkinson, I. E. Portugues, P. J. Moore, and R. Rutherford, "Impulsive noise modeling and prediction of its impact on the performance of WLAN receiver," IEEE 17th European Signal Processing Conference, Aug. 2009.

[5] B. Zhu, Z. Zeng, and J. Cheng, "Arbitrarily tight bounds on cumulative distribution function of beckmann distribution," International conference on computing networking and communications, pp. 41-45, Jan. 2017. DOI:10. 1109/ICCNC.2017.7876099 


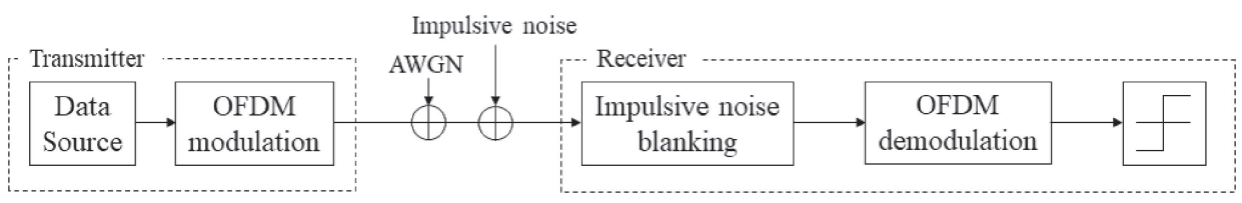

Fig. 1. OFDM-PLC system model.

\section{Introduction}

To enable high speed home networks, power-line communication (PLC) has been widely investigated [1]. Since electric powerline is not designed for wideband signal transmissions, the received signal is severely distorted due to dispersive channel characteristics. To mitigate the signal distortion, multi-carrier transmission techniques such as orthogonal frequency division multiplexing (OFDM) are used for data transmission over power-line [2]. In PLC systems, mitigation of impulsive noise is important to enhance the transmission performance further [3]. One of the existing approaches is to apply a deliberate blanking to the received signal for mitigating impulsive noise. In this scheme, the received samples are removed whenever they exceed a given threshold and consequently impulsive noise is effectively suppressed if the signal amplitude is below the blanking threshold. However, the received signal exceeds the blanking threshold, missdetection of impulsive noise may occur at the receiver and consequently degrade bit error rate (BER) performance due to erroneous blanking. To reduce missdetection of impulsive noise, it is important to determine the blanking threshold properly. Although the probability of false alarm (PoF) when blanking is applied to the OFDM system is discussed in [3], detailed analysis on the probability of detection (PoD) and the influence of blanking on BER are not given.

In this article, we theoretically analyze the impact of the blanking threshold selection on achievable performance, i.e., PoD, PoF, and BER in presence of impulsive noise. Based on the results, we discuss the impact of blanking threshold selection on detection accuracy of impulsive noise and achievable BER at the receiver.

\section{System model}

Fig. 1 shows block diagram of OFDM-PLC system considered in this study. On the transmitter side, OFDM modulation is implemented with inverse fast Fourier transform (IFFT). The received signal is affected by additive white Gaussian noise (AWGN) and impulsive noise, where Middleton's Class-A impulsive noise model [4] is used. Complex Class-A noise $n(t)$ is expressed as

$$
n(t)=w(t)+i(t),
$$

where $w(t)=w_{I}(t)+j w_{Q}(t)$ and $i(t)=i_{I}(t)+j i_{Q}(t)$ denote complex AWGN and complex impulsive noise terms, respectively. In this model, probability of occurrence of impulsive noise follows a Poisson distribution, while probability density function (PDF) of impulsive noise amplitude follows a Gaussian distribution with zero-mean and variance $\sigma_{i}^{2} / \lambda$. Here, $\sigma_{i}^{2}$ and $\lambda$ denote the variance of the distribution of $i_{I}(t)$ (or $i_{Q}(t)$ ) and the number of impulsive noise occurrences per unit-time, 
respectively. According to property of Poison distribution, we assume that impulsive noise never occurs at both real part and imaginary part, simultaneously (i.e., $\left.i_{I}(t) i_{Q}(t)=0\right)^{1}$. Let $\sigma_{w}^{2}$ denotes the variance of the distribution of $w_{I}(t)$ (or $w_{Q}(t)$ ). Here, $\Gamma=\sigma_{w}^{2} / \sigma_{i}^{2}$. Note that higher impulsive noise appears when $\Gamma$ is smaller. Assuming that probability of occurrence of impulsive noise follows a Poisson distribution with mean value of $\Lambda=\lambda / N_{s}$, the PDF of Class-A noise amplitude $\eta=|n(t)|$ is given as follows [4]:

$$
p_{c}(\eta)=e^{-\lambda} \sum_{k=0}^{\infty} \frac{\lambda^{k}}{k ! \sqrt{2 \pi \sigma_{k}^{2}}} \exp \left(-\frac{\eta^{2}}{2 \sigma_{k}^{2}}\right),
$$

where $N_{s}$ denotes the number of samples per OFDM symbol and $\sigma_{k}^{2}=\frac{k / \lambda+\Gamma}{1+\Gamma}$. In Eq. (1), as $\lambda$ increases, the probability of impulsive noise occurrence increases while magnitude of impulsive noise decreases.

At the receiver, if magnitude of the received signal amplitude exceeds a given blanking threshold $\beta$, the deliberate blanking is adopted as

$$
\hat{r}(t)= \begin{cases}0, & |r(t)|>\beta \\ r(t), & |r(t)| \leq \beta,\end{cases}
$$

where $r(t)$ and $\hat{r}(t)$ denote the input and its output complex signal at the blanking, respectively. After that, OFDM demodulation is carried out with fast Fourier transform (FFT) to detect the data.

\section{Performance analysis of OFDM-PLC system with blanking}

\subsection{Probability of detection (PoD) of impulsive noise}

$\mathrm{PoD}$ of impulsive noise is defined as a probability that an impulsive noise is correctly detected when it exists. Let $x(m \Delta T)=x_{I}(m \Delta T)+j x_{Q}(m \Delta T)$ denotes complex OFDM signal at $t=m \Delta T$ whose real and imaginary parts are $x_{I}(m \Delta T)$ and $x_{Q}(m \Delta T)$, respectively. Here, $\Delta T$ is sampling interval. Hereafter, for simplicity of notations, we omit index $m$. Assume that PDFs of $x_{I}$ and $x_{Q}$ follow Gaussian distribution, i.e.,

$$
p_{g}\left(x_{I}\right)=\frac{1}{\sqrt{2 \pi \sigma_{x}^{2}}} \exp \left(-\frac{x_{I}^{2}}{2 \sigma_{x}^{2}}\right) \text { and } p_{g}\left(x_{Q}\right)=\frac{1}{\sqrt{2 \pi \sigma_{x}^{2}}} \exp \left(-\frac{x_{Q}^{2}}{2 \sigma_{x}^{2}}\right),
$$

where $\sigma_{x}^{2}$ denotes variance of random variable $x_{I}$ and $x_{Q}$. PDF of the absolute amplitude (i.e., $|x|=\sqrt{x_{I}^{2}+x_{Q}^{2}}$ ) follows Rayleigh distribution as

$$
p_{x}(|x|)=\frac{|x|}{\sigma_{x}^{2}} \exp \left(-\frac{|x|^{2}}{2 \sigma_{x}^{2}}\right) .
$$

Let $w=w_{I}+j w_{Q}$ denotes complex AWGN, where $w_{I}$ and $w_{Q}$ are random variables which follow the same Gaussian distribution with variance $\sigma_{w}^{2}$. As explained in the previous section, we assume impulsive noise occurs at either real or imaginary part. Based on this property, let us consider impulsive noise occurs only at the real part (i.e., $i_{Q}=0$ ). Thus, the received complex signal can be expressed as

${ }^{1}$ Since either $i_{I}(t)$ or $i_{Q}(t)$ is always zero (i.e., $\left.i_{I}(t) i_{Q}(t)=0\right)$, statistical distribution of complex amplitude of impulsive noise $|i(t)|$ is the same as those of $\left|i_{I}(t)\right|$ and $\left|i_{Q}(t)\right|$, respectively. Thus, PDF of impulsive noise amplitude follows Gaussian distribution. 


$$
\begin{aligned}
r & =x+w+i_{I} \\
& =\left(x_{I}+w_{I}+i_{I}\right)+j\left(x_{Q}+w_{Q}\right),
\end{aligned}
$$

where $x_{I}\left(x_{Q}\right), w_{I}\left(w_{Q}\right)$, and $i_{I}$ are random variables which follow Gaussian distribution with variances $\sigma_{x}^{2}, \sigma_{w}^{2}$, and $\sigma_{i}^{2}$, respectively. Hence, variance of real part distribution of $r$ is $\sigma_{1}^{2}=\sigma_{x}^{2}+\sigma_{w}^{2}+\sigma_{i}^{2}$ and that of imaginary part is $\sigma_{2}^{2}=$ $\sigma_{x}^{2}+\sigma_{w}^{2}$, respectively. When both real and imaginary part are Gaussian random variable but their variances are different (i.e., $\sigma_{1}^{2}$ and $\sigma_{2}^{2}$ ), PDF of received signal amplitude $|r|$ is given as follows [5]:

$$
\begin{aligned}
p_{z}(|r|)= & \frac{|r|}{2 \pi \sigma_{1} \sigma_{2}} \\
& \times \int_{0}^{2 \pi} \exp \left[-\frac{(|r| \cos \theta)^{2}}{2 \sigma_{1}^{2}}-\frac{(|r| \sin \theta)^{2}}{2 \sigma_{2}^{2}}\right] d \theta .
\end{aligned}
$$

From Eq. (6), probability of impulsive nose detection (PoD) is given as a probability that $|r|$ exceeds a certain threshold $\beta$ as

$$
P_{d}(\beta)=\int_{\beta}^{\infty} p_{z}(r) d r .
$$

\subsection{Probability of false alarm (PoF) of impulsive noise}

PoF of impulsive noise is defined as a probability that impulsive noise is erroneously detected at time instance when impulsive noise does not exist. The status of the received complex signal $r$ is expressed as the following binary hypothesis:

$$
r= \begin{cases}x+w+i & \mathcal{H} \\ x+w & \overline{\mathcal{H}}\end{cases}
$$

where $\mathcal{H}$ and $\overline{\mathcal{H}}$ denote cases that impulsive noise exists and its opposite, respectively. Probability of $\mathcal{H}$ occurrence is given as $P(\mathcal{H})=p / N_{s}$ and hence the opposite is given as $P(\overline{\mathcal{H}})=1-P(\mathcal{H})$, where $p\left(0 \leq p \leq N_{s}\right)$ is random variable that follows Poison distribution with non-negative average value $\lambda\left(0<\lambda \ll N_{S}\right)$. In the absence of impulsive noise, according to Bayes' theorem, PoF is given as a function of the blanking threshold $\beta$ as follows:

$$
P_{f}(\beta)=P(|r|>\beta, \overline{\mathcal{H}})=P(\overline{\mathcal{H}}) P(|r|>\beta \mid \overline{\mathcal{H}}) .
$$

In fact, when $x_{I}$ and $w_{I}$ follow Gaussian distribution with zero-mean and variance $\sigma_{x}^{2}$ and $\sigma_{w}^{2}$, real and imaginary parts of $r=x+w$ also follow Gaussian distribution with zero-mean and variance $\sigma_{x}^{2}+\sigma_{w}^{2}$. Hence, $|r|$ follows the following Rayleigh distribution:

$$
p_{r}(|r|)=\frac{|r|}{\sigma_{x}^{2}+\sigma_{w}^{2}} \exp \left(-\frac{|r|^{2}}{2\left(\sigma_{x}^{2}+\sigma_{w}^{2}\right)}\right) .
$$

From Eq. (9) and Eq. (10), we can obtain

$$
\begin{aligned}
P_{f}(p, \beta) & =P(|r|>\beta, \overline{\mathcal{H}}) \\
& =P(\overline{\mathcal{H}}) P(|r|>\beta \mid \overline{\mathcal{H}})
\end{aligned}
$$




$$
\begin{aligned}
& =\left(1-\frac{p}{N_{s}}\right) \underbrace{\int_{\beta}^{\infty} \frac{r}{\sigma_{x}^{2}+\sigma_{w}^{2}} \exp \left(-\frac{r^{2}}{2\left(\sigma_{x}^{2}+\sigma_{w}^{2}\right)}\right) d r}_{P(|r|>\beta \mid \overline{\mathcal{H}})} \\
& =\left(1-\frac{p}{N_{s}}\right) \exp \left(-\frac{\beta^{2}}{2\left(\sigma_{x}^{2}+\sigma_{w}^{2}\right)}\right) .
\end{aligned}
$$

Hence, average PoF is given as

$$
\begin{aligned}
\overline{P_{f}}(\beta) & =\sum_{p=0}^{\infty}\left[\frac{e^{-\lambda} \lambda^{p}}{p !} P_{f}(p, \beta)\right] \\
& =\sum_{p=0}^{\infty}\left[\frac{e^{-\lambda} \lambda^{p}}{p !} \exp \left(-\frac{\beta^{2}}{2\left(\sigma_{x}^{2}+\sigma_{w}^{2}\right)}\right)\left(1-\frac{p}{N_{s}}\right)\right] .
\end{aligned}
$$

\section{BER of OFDM with blanking}

Received complex signal at the $l$-th subcarrier in the $s$-th OFDM symbol $r^{(l)}[s]=r_{I}^{(l)}[s]+j r_{Q}^{(l)}[s]$ is given as

$$
r^{(l)}[s]=x^{(l)}[s]+n^{(l)}[s]+d^{(l)}[s],
$$

where $x^{(l)}[s]=x_{I}^{(l)}[s]+j x_{Q}^{(l)}[s]$ is the complex transmit signal at the $l$-th subcarrier in the $s$-th OFDM symbol. $n^{(l)}[s]=n_{I}^{(l)}[s]+j n_{Q}^{(l)}[s]$ and $d^{(l)}[s]=d_{I}^{(l)}[s]+j d_{Q}^{(l)}[s]$ represent the complex Class-A noise component and the complex distortion signal by blanking operation observed at the $l$-th subcarrier in the $s$-th OFDM symbol. Hereafter, for simplicity of notations, we omit symbol index $s$. Since real part and imaginary part of the received signal have the same statistical characteristics, we consider only real part of $y^{(l)}$ in the following discussion. To analyze BER of OFDM with blanking in presence of Class-A noise, we assume that PDF of $d_{I}^{(l)}$ follows Gaussian distribution with mean value $\mu_{(\gamma, \beta)}$ and variance $\sigma_{(\gamma, \beta)}^{2}$, where $\gamma$ represents bit energy $\left(E_{b}\right)$ to noise power density $\left(N_{0}\right)$ ratio (i.e., $\left.E_{b} / N_{0}\right) \cdot \mu_{(\gamma, \beta)}$ and $\sigma_{(\gamma, \beta)}^{2}$ are changed depending on $\gamma$ and $\beta$. In this study, statistical parameters, $\mu_{(\gamma, \beta)}$ and $\sigma_{(\gamma, \beta)}^{2}$ are determined by simulation. From this assumption, $n_{I}^{(l)}+d_{I}^{(l)}$ also follows Gaussian distribution with mean value $\mu_{(\gamma, \beta)}$ and variance $\sigma_{(\gamma, \beta)}^{2}+\sigma_{n}^{2}$, because $n_{I}^{(l)}$ follows Gaussian distribution with zero-mean and variance $\sigma_{n}^{2}$. PDF of $d_{I}^{(l)}+n_{I}^{(l)}$ is given as

$$
p_{b}\left(d_{I}^{(l)}+n_{I}^{(l)}, \gamma, \beta\right)=\frac{1}{\sqrt{2 \pi\left(\sigma_{(\gamma, \beta)}^{2}+\sigma_{n}^{2}\right)}} \exp \left(-\frac{\left(\left(d_{I}^{(l)}+n_{I}^{(l)}\right)-\mu_{(\gamma, \beta)}\right)^{2}}{2\left(\sigma_{(\gamma, \beta)}^{2}+\sigma_{n}^{2}\right)}\right) .
$$

Let us assume quadrature phase shift keying (QPSK) modulation with amplitude $A$ or $-A$ at each subcarrier. Then, by integrating Eq. (14) with respect to $\xi=$ $d_{I}^{(l)}+n_{I}^{(l)}$, BER of QPSK-OFDM with blanking is obtained as

$$
P_{e}(\gamma, \beta)=\frac{1}{\sqrt{2 \pi\left(\sigma_{(\gamma, \beta)}^{2}+\sigma_{n}^{2}\right)}} \int_{-\infty}^{0} \exp \left(-\frac{\left(\xi-\left(A+\mu_{(\gamma, \beta)}\right)\right)^{2}}{2\left(\sigma_{(\gamma, \beta)}^{2}+\sigma_{n}^{2}\right)}\right) d \xi .
$$




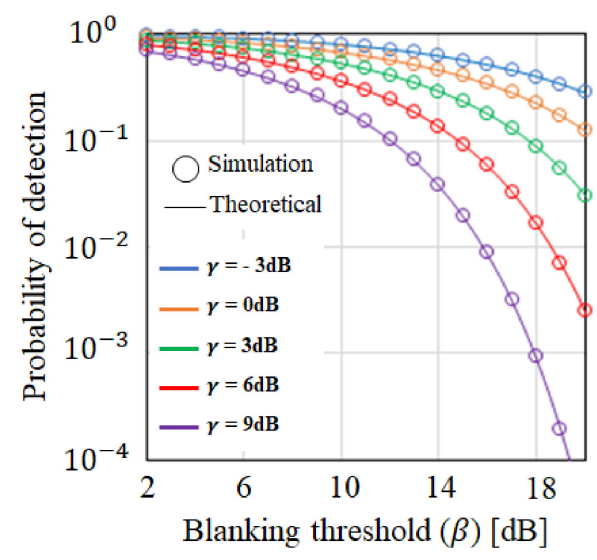

(a) Probability of detection.

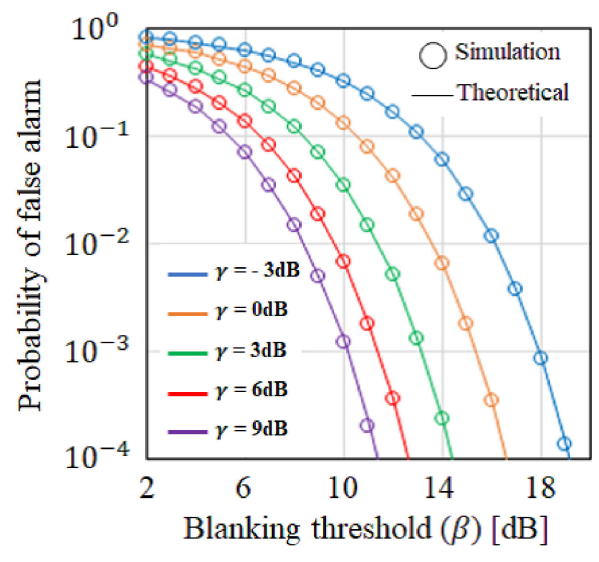

(b) Probability of false alarm.

Fig. 2. PoD and PoF of impulsive noise.

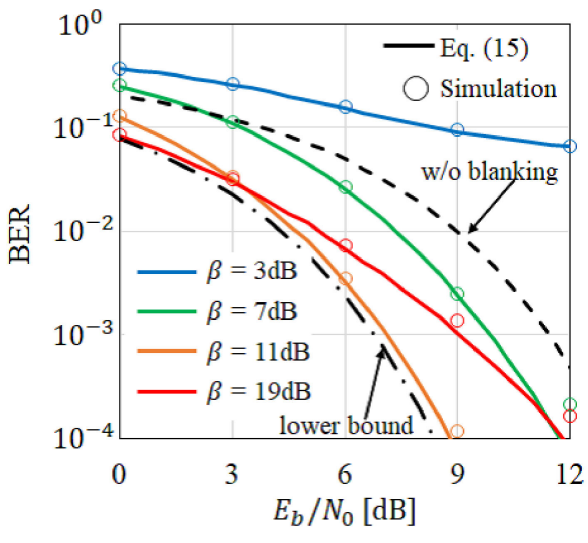

(a) $E_{b} / N_{o}$ vs BER.

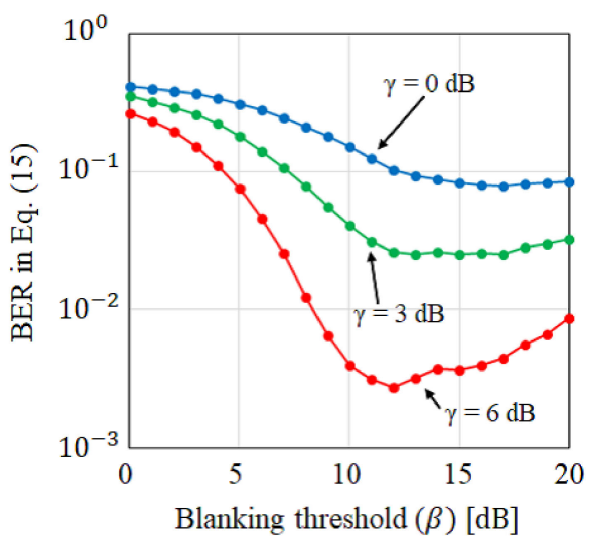

(b) $\beta$ vs BER in Eq. (15).

Fig. 3. BER performance of OFDM with blanking.

\section{Performance evaluation}

To clarify the validity of analysis in Sect. 3, we compare expressions in Eqs. (7), (12), and (15) with the corresponding simulation results. System model is the same as in Fig. 1. Parameters of Class-A noise are set to $\Gamma=0.001$ and $\Lambda=0.01$, respectively. The number of subcarriers of OFDM signal is 64 and $N_{s}=512$ is used.

Fig. 2 shows theoretical expressions of $\mathrm{PoD}$ and PoF in Eqs. (7) and (12) as a function of impulsive noise blanking threshold $\beta$, where $\gamma=E_{b} / N_{0}$ are set to $-3,0$, 3,6 , and $9 \mathrm{~dB}$. For comparison, simulation results in the same condition are also plotted. The results prove that theoretical expression of PoD and PoF show good agreement with the simulation results.

Fig. 3(a) shows BER of OFDM system with blanking technique, where QPSK is used for subcarrier modulation scheme. In this figure, BER in Eq. (15) is plotted as solid line. The corresponding simulation results are also plotted. Blanking threshold $\beta$ is set to $3,7,11$, and $19 \mathrm{~dB}$. The label "lower bound" illustrates the case where all impulsive noise is removed perfectly on the receiver side without distorting the received signal. We can confirm that BER in Eq. (15) has good agreement with its simulation results. This implies that distortion due to 
the blanking can be approximated as random variable which follows Gaussian distribution.

Fig. 3(b) shows the relation between blanking threshold $\beta$ and BER in Eq. (15), where $\gamma$ are set to 0,3 and $6 \mathrm{~dB}$ and $\beta$ takes discrete values from 0 to $20 \mathrm{~dB}$. From this figure, in the case of $\gamma=0 \mathrm{~dB}$, BER is minimized at $\beta=17 \mathrm{~dB}$. On the other hand, in case of $\gamma=6 \mathrm{~dB}$, BER is minimized at $\beta=12 \mathrm{~dB}$. This is because magnitude of impulsive noise is reduced as $\gamma=E_{b} / N_{0}$ increases and thus the optimum blanking threshold depends on $\gamma$. These results imply that the blanking threshold needs to be determined according to $\gamma$ to improve the PoD while keeping the PoF below a required value.

\section{Conclusion}

In this article, we have analyzed probability of impulsive noise detection (PoD) on the receiver side and probability of its false alarm (PoF). The analyzed results show good agreements with the simulation results. In addition, we have evaluated BER performance of OFDM with deliberate blanking based on the assumption that PDF of the received signal amplitude at each subcarrier follows Gaussian distribution.

\section{Acknowledgments}

This research was partially supported by the JSPS KAKENHI (JP17K06427, JP17J04710), and the Telecommunications Advancement Foundation. 